\title{
1 A longitudinal study of white matter 2 functional network in mild traumatic brain 3 injury
}

4 Xiaoyan $\mathrm{Jia}^{1 \#}$, Xuebin Chang ${ }^{3 \#}$, Lijun Bai ${ }^{1,2 \#}$, Yulin Wang ${ }^{4,5}$, Debo Dong ${ }^{3,6}$, Shuoqiu Gan ${ }^{1}$, Shan

$5 \quad \mathrm{Wang}^{1}$, Xuan $\mathrm{Li}^{1}$, Xuefei Yang ${ }^{1}$, Yinxiang Sun ${ }^{7}$, Tianhui $\mathrm{Li}^{1}$, Feng Xiong ${ }^{1}$, Xuan Niu ${ }^{7}$, Hao Yan ${ }^{8} 9^{*}$

6

$7{ }^{1}$ The Key Laboratory of Biomedical Information Engineering, Ministry of Education, Department of 8 Biomedical Engineering, School of Life Science and Technology, Xi'an Jiaotong University, Xi'an 9710049 , China

$10{ }^{2}$ Department of Radiology, the Second Affiliated Hospital and Yuying Children's Hospital of 11 Wenzhou Medical University, Wenzhou 325027, China

$12{ }^{3}$ The Clinical Hospital of Chengdu Brain Science Institute, MOE Key Lab for Neuroinformation, 13 Center for Information in Medicine, High-Field Magnetic Resonance Brain Imaging Key Laboratory of 14 Sichuan Province, School of Life Science and Technology, University of Electronic Science and

15 Technology of China, Chengdu 611731, China

$16{ }^{4}$ Department of Experimental and Applied Psychology, Faculty of Psychological and Educational 17 Sciences, Vrije Universiteit Brussel, Brussels 1040, Belgium

$18{ }^{5}$ Department of Data Analysis, Faculty of Psychology and Educational Sciences, Ghent University, 19 Henri Dunantlaan 1, Ghent B-9000, Belgium

$20{ }^{6}$ Institute of Neuroscience and Medicine, Brain \& Behaviour (INM-7), Research Centre Jülich, 21 Jülich, Germany

$22{ }^{7}$ Department of Medical Imaging, the First Affiliated Hospital of Xi' an Jiaotong University, Xi'an,

23 China

$24{ }^{8}$ Key Laboratory for artificial intelligence and cognitive neuroscience of language, Xi' an International 25 Studies University, Xi' an 710128, China 
bioRxiv preprint doi: https://doi.org/10.1101/2020.09.25.313338; this version posted September 28, 2020. The copyright holder for this preprint (which was not certified by peer review) is the author/funder, who has granted bioRxiv a license to display the preprint in perpetuity. It is made available under aCC-BY-ND 4.0 International license.

$1{ }^{9}$ Department of linguistics, School of foreign languages, Xidian University, Xi' an 710126, China

2

$3{ }^{\#}$ These authors contributed equally to this study.

4 "Correspondence: Hao Yan

$5 \quad$ E-mail: xiaoyan_jia@stu.xjtu.edu.cn

6 Address: Key laboratory for artificial intelligence and cognitive neuroscience of language, Xi'an

7 International Studies University, Xi' an 710128, China 


\section{Abstract}

2 The mild traumatic brain injury (mTBI) results in traumatic axonal injury, which damages the

3 long-distance white matter (WM) connections and thus disrupts the functional connectome of

4 large-scale brain networks that support cognitive function. Patterns of WM structural damage following

5 mTBI were well documented using diffusion tensor imaging, however, the functional organization of

$6 \mathrm{WM}$ and its association with grey matter functional networks (GM-FNs) and cognitive assessments

7 remains unknown. The present study adopted resting-state functional magnetic resonance imaging to

8 explore WM functional properties in mTBI patients (113 acute patients, 56 chronic patients, 47 healthy

9 controls (HCs)). Eleven large-scale WM functional networks (WM-FNs) were constructed by the

10 k-means clustering algorithm which carried out in voxel-wise WM functional connectivity (FC).

11 Compared to HCs, acute mTBI patients showed enhanced FC between inferior fronto-occipital

12 fasciculus (IFOF) WM-FN and primary sensorimotor WM-FNs, and cortical primary sensorimotor

13 GM-FNs. And FC between IFOF WM-FN and anterior cerebellar GM-FN was positively correlated

14 with information processing speed. Moreover, all of these WM-FNs abnormalities were returned to the

15 normal level at the chronic stage. Our findings suggest the compensatory mechanism of cognitive

16 deficits in the acute stage and its involvement in facilitating recovery from cognitive deficits in the

17 chronic stage. The convergent damage of the IFOF network highlighted its key role in our

18 understanding of the pathophysiology mechanism of mTBI patients and thus might be regarded as a

19 biomarker in the acute stage and a potential indicator of treatment effect.

20 Key words: Mild traumatic brain injury, White matter functional networks, fMRI, Resting-state 21 functional connectivity, Compensatory mechanism. 


\section{Introduction}

2 Traumatic brain injury (TBI) is a public health challenge of vast proportions but insufficiently

3 recognized. More than 50 million people have a TBI worldwide each year, and it is evaluated that

4 about half of the world's population will have one or more TBIs in their lifetime [1,2].

5 The mild TBI (mTBI) accounts for 80\% 90\% of all TBI cases in both civilian and military populations

6 [3]. In general, mTBI results in traumatic axonal injury [4,5]. Previous studies suggested that the

7 traumatic axonal injury would induce cortical network disconnection and disrupt cortical-subcortical

8 pathways in mTBI [6-8], which further impair information transfer across brain networks [9] and

9 produce cognition deficits [10]. Importantly, the long white matter (WM) tracts connections are

10 particularly vulnerable to the mechanical trauma of TBI [11] and thus disrupt the functional

11 communication of large-scale networks that support cognition [12]. For instance, the inferior

12 fronto-occipital fasciculus (IFOF) is a brain region mediates feed-forward propagation of visual input

13 to anterior frontal regions and was found to have direct top-down modulation of early visual processing

14 [13]. IFOF was documented with reduced WM integrity in mTBI patients [14]. Besides, the corpus

15 callosum and internal capsule are regions where projection and contact fibers are highly concentrated.

16 These two regions are responsible for the transmission of important information such as somatosensory

17 and motor signals, and are the most commonly damaged regions in mTBI $[15,16]$. And previous study

18 evidenced the cognition deficit might due to the damage of WM connecting the widely distributed grey matter (GM) networks [17], suggested the WM play a crucial role in information communication

20 among large-scale brain networks. Although previous studies revealed the alterations in WM tracts in 21 mTBI patients using diffusion tensor imaging (DTI), functional organization of WM and its association

22

23

24

25

26

27 with GM functional networks (GM-FNs) across whole brain remains unknown.

Over the past two-decades, resting-state functional magnetic resonance imaging (rs-fMRI) has been a powerful technique for investigating the functional architecture of human GM $[18,19]$. Recently, increasing studies have evidenced the existence of functional information in WM, which can also be detected by rs-fMRI [20-22]. For instance, Ding et al. uncovered that resting-state BOLD signals in WM reflect neural coding and information processing [20]. Several studies further indicated that the 
1 WM would be activated in response to multiple tasks, such as perceptual and motor tasks [23-25].

2 Besides, the large-scale functional organization of WM has been built [26,27]. Furthermore, recent

3 studies revealed that the functional connectivity (FC) in WM is related to the underlying

4 pathophysiological mechanism of neuropsychiatric disorders, such as Parkinson's disease [28],

5 schizophrenia [29], and epilepsy [30]. Together, all previous studies have suggested the involvement of

6 functional network of WM in cognitive and disorder progression. Evidently, the WM lesions widely

7 exist in mTBI and the impact of these lesions on cognition is well documented in previous studies.

8 Therefore, the present study constructed the WM-FNs of mTBI patients, and further investigated the

9 longitudinal neuroimaging trajectories of functional properties of WM functional networks (WM-FNs)

10 and its association with GM-FNs in mTBI patients to deepen our understanding of the pathological

11 mechanism of cognitive deficits in mTBI patients.

12 The present study aims to (i) construct large-scale WM-FNs by k-means clustering analysis on rs-fMRI

13 data of a large cohort of 113 acute mTBI patients (of which 56 patients followed-up at six months to

14 one year, which were considered as chronic stage patients) and 47 healthy controls (HCs). (ii)

15 investigate the FC within the resulting large-scale WM-FNs and its interaction with the known

16 GM-FNs in both acute and chronic mTBI patients. (iii) estimate the spatial interaction between the 17 resulting WM-FNs and structural WM tracts. (iv) measure the correlations between WM functional 18 disturbances and cognitive and clinical assessments of mTBI patients.

\section{Material and methods}

\section{Participants}

21 One hundred and thirteen consecutive patients suffering from head trauma were recruited from the

22 local emergency department between August 2016 and May 2018 as the initial population. The

23 inclusion and exclusion criteria of mTBI were based on the World Health Organization's Collaboration

24 Centre for Neurotrauma Task Force [31]. The detailed inclusion and exclusion criteria of mTBI

25 patients were described in the supplementary material. Fifty-six of mTBI patients in the acute stage 
1 were followed up for six months to one year, with repeat MRI and cognitive and clinical assessment.

2 Forty-seven age-, gender-, and education-matched HCs with no history of neurologic impairment or

3 psychiatric disorders were included.

4 MRI scans of patients with mTBI were initially assessed in acute stage (within 7 days post-injury) and

5 followed up at chronic stage (6 months to 1-year post-injury). Cognitive and clinical assessment (see

6 below) was carried out within 48 hours of MRI scans. All HCs participants also underwent an identical

$7 \quad$ MRI scanning and cognitive and clinical assessment.

8 All participants were right-handed according to the Edinburgh Handedness Inventory [32]. Written

9 informed consent was obtained from each individual before the experimental procedures. The research

10 procedures have been approved by the Local Institutional Review Board (the First Affiliated Hospital

11 of Xi' an Jiaotong University) and conducted in line with the Declaration of Helsinki.

\section{Image acquisition}

13 A non-contrast CT scan was performed on all consecutive patients following acute head injury with a 14 64-row CT scanner (GE, Lightspeed VCT). All participants underwent MRI scanning in a $3.0 \mathrm{~T}$ MRI 15 scanner (GE 750) with a 32-channel head coil. The detailed scan parameters were described in the 16 supplementary material.

\section{Cognitive and clinical assessment}

18 Cognitive assessment included: (a) Trail-Making Test Part A (TMT-A) and WAIS-III Digital Symbol

19 Coding score (DSC), which were used to evaluate cognitive information processing speed [33]; (b)

20 WAIS-III Forward Digit Span (DS) and Backward DS, which were used to measure working memory

21 [34]; (c) Verbal Fluency (VF) Test, which was used to examine language ability, semantic memory,

22 and executive function [35]. And clinical assessment included: (a) Post-concussive symptoms were

23 evaluated based on Rivermead Post-Concussion Symptom Questionnaire (RPCS) [36]; (b) Insomnia

24 Severity Index (ISI) [37]; (c) Posttraumatic stress disorder (PTSD) Checklist-Civilian Version (PCL-C)

$25[38]$. 


\section{Data Preprocessing}

2 Preprocessing steps of rs-fMRI and T1 data were carried out using the DPABI (http://rfmri.org/dpabi)

3 [39] and SPM (https://www.fil.ion.ucl.ac.uk/spm). Fig. 1 demonstrated the preprocessing steps. In

4 detail, the rs-fMRI data were 1) discarded the first five volumes, 2) slice-time corrected, 3) realigned, 4)

5 regressed out linear trend signal, 24 head motion parameters, and the mean cerebrospinal fluid (CSF)

6 signals, 5) performed temporal scrubbing using motion "spike" (framewise displacement $($ FD) $>0.5)$ as

7 separate repressors, 6) filtered by using a band-pass filter $(0.01$ to $0.15 \mathrm{~Hz})$ for reducing the

8 non-neuronal contribution to BOLD signals which is in line with prior WM FC studies $[27,29], 7)$

9 spatial smoothed (full width at half maximum $=4 \mathrm{~mm}$ ) of WM and GM signals separately within

10 individual WM mask or GM mask to avoid the mixture of WM and GM signals, 8) normalized to

11 standard EPI template and resampled voxel size into $3 \mathrm{~mm} \times 3 \mathrm{~mm} \times 3 \mathrm{~mm}$. For T1 image, the T1 data

12 were segmented into WM, GM, and CSF using SPM's New Segment algorithm. The T1 segmentation

13 results from individual subjects were co-registered to the functional data space for each individual for

14 further the identification WM or GM mask (the segmentation threshold was set to 0.5 using SPM's

15 tissue segmentation).

16 Preprocessing of DTI data was performed using FSL (https://fsl.fmrib.ox.ac.uk/fsl) software [40], the

17 preprocessing steps included skull stripping of the b0 image, eddy current correction, head motion

18 correction, and registering and aligning to T1 image by mutual information algorithm. The DTI and

19 anatomical T1 images were normalized into MNI space.

\section{Clustering WM-FNs}

21 The processed resting-state data of mTBI patients in the acute stage and HCs subjects were used to 22 cluster WM-FNs.

23 First, the segmentation results about the T1 image of each individual were used to create the group

24 WM and GM mask (Fig. 1). For each voxel of each subject, the identification of WM, GM, or CSF was

25 depended on the maximum probability value of the T1 segmentation results. The WM, GM, and CSF 
1 mask for each individual were generated subsequently. And the percentage of participants classified as

2 WM or GM was obtained by averaging across all participants. For WM, voxel with a percentage

3 greater than $60 \%$ of subjects was identified as a group-level WM mask. For GM, a loose threshold of a

4 percentage greater than $20 \%$ of subjects was identified as a group-level GM mask but excluded the

5 voxels included in the WM mask. Of note, to correctly classify deep brain structures [41], the

6 subcortical areas (according to the Harvard-Oxford Atlas [42]) were excluded from the WM mask.

7 Besides, the resulting masks were used to compare with the preprocessed functional data, then we

8 excluded voxels that were identified as GM or WM but without functional data in $>20 \%$ of the subjects.

9 The group-level WM mask was further co-registered to the functional data space and resampled for

10 rs-fMRI processing. The group-level WM mask contained 17863 voxels.

11 After generating the GM and WM mask, the K-means clustering algorithm on the averaged group-level

12

13 resting-state correlation matrices was used to obtain WM-FNs (Fig. 1). To reduce computational complexity, the 17863 voxels of the WM mask were subsampled into 4466 nodes using an interchanging grid strategy [43]. Specifically, any second voxel was extracted along the rows and columns and then moved one between the two slices. The Pearson correlation matrix was calculated between all WM voxels and sub-sample nodes to obtain the correlation matrix $(17863 \times 4466)$ for each subject. K-means clustering method (distance metric-correlation, 10 replicates) from machine learning was carried out in the mean correlation matrices. Given clustering method was carried out for an unequal number between patients and the HCs group, the clustering results have the possibility to focus more on the patients, thus lead to the results more inclined to patients. Therefore, the correlation matrix was first averaged in HCs group and mTBI group separately, and then averaged again to obtain final correlation matrix which used for clustering.

Furthermore, to identify the most reliable number of WM-FNs, the stability of each number of clusters ranging from 2 to 22 was evaluated. The whole connectivity matrix $(17863 \times 4466)$ was randomly divided into four sub-folds $(17863 \times 1125)$. For each kinds of cluster number, each sub-fold carried out the same clustering computation separately so that four clustering results were obtained. To evaluate the similarity of clustering between different sub-folds, the adjacency matrix for each sub-fold was calculated. Then these adjacency matrices were compared using Dice's coefficient. The stability of the 
1 number of clusters was evaluated according to the averaged coefficient of Dice. Furthermore, to ensure

2 the stability of the above results, the above process was repeated for 10 times. Afterward, the average

3 Dice's coefficient value of the 10 times was taken as the final Dice's coefficient.

4 The WM-FNs atlas at the acute stage was also used to analyze the pathological changes of WM-FNs in

5 the chronic stage. To avoid the alteration of the results by the functional reconstruction of WM-FNs in

6 the chronic stage, the WM-FNs clustering of the chronic stage were added (see Supplementary

7 material).

\section{Similarity between WM-FNs and DTI fiber tracts}

9 The spatial similarity between the WM-FN and DTI fiber tracts was evaluated to investigate the spatial 10 correspondence between them. To identify DTI tracts, the automated fiber quantification algorithm was 11 used to automatically identify 20 fiber tracts depended on the JHU WM tractography atlas. When the voxels where a DTI fiber tract identified in > 2 subjects and belonged to the group-level WM mask

13 constructed previously, the voxels were incorporated into the DTI fiber tract. It should be noted that 14 symmetrical DTI fiber tracts in two hemispheres of the brain were combined as one tract. Furthermore, 15 to investigate the similarity between WM-FNs and DTI fiber tracts, the number of overlapping voxels 16 of each WM-FN and each DTI fiber tract were calculated. The percentage of voxels in the WM-FN 17 which can be identified as part of the DTI tracts (overlap/WM-FN) was also calculated.

\section{Functional connectivity}

19 To evaluate the interaction within the resulting WM-FNs, the average signal time-courses from

20 WM-FNs were extracted by averaging the signal values across all voxels which were belonged to each

21 network for each individual. The Pearson's correlation between any two WM-FNs' time-courses for each individual was computed. Besides, to evaluate the interaction between the WM-FNs and GM-FNs,

23 the GM-FNs atlas which was generated by the same clustering procedure [44] was used, and Pearson's

24 correlation between the WM-FNs and GM-FNs was also computed. These correlation coefficients were 25 averaged across subjects to obtain a group level matrix representing the relationship between WM and 
1 GM-FN. Of note, the Pearson's correlation coefficients were transformed into the Fisher z score before

2 statistical analysis.

\section{Statistical analysis}

4 The normality distribution of all cognitive and clinical continuous variables was tested by the

5 Shapiro-Wilk W test. The group differences about cognitive and clinical continuous variables were

6 calculated by the independent two-sample t-test and Mann-Whitney test based on data normality,

7 respectively. Chi-square was used to compare cognitive and clinical categorical variables, with a

8 significant set at $\mathrm{p}<0.05$.

9 And independent two-sample t-test was used to show the differences between the mTBI and HCs about

10 the z-score of FC (Pearson's correlation coefficient), with a significance set at $\mathrm{p}<0.05$ (false discovery

11 rate corrected, FDR corrected). Since the age of chronic stage patients and HCs was not very well

12 matched $(\mathrm{p}=0.064)$, age was regressed in the statistical analysis of the chronic stage to avoid the

13 influence of brain development.

\section{Correlation between altered WM-FNs and cognitive and clinical}

\section{5 assessments}

16 To evaluate the relationship between altered imaging results and cognitive and clinical assessments, we

17 calculated the Spearman correlation between altered FC and cognitive and clinical variables since the

18 cognitive and clinical variables were not normally distributed.

\section{Results}

\section{Demographic, cognitive and clinical characteristics}

21 Sixteen patients with mTBI in the acute stage and four HCs with head motion scans exceeding $2 \mathrm{~mm}$

22 and/or $2^{\circ}$ rotation were excluded. The final analysis included $97 \mathrm{mTBI}$ patients in the acute stage, 56 
1 mTBI patients in the chronic stage, and 43 HCs. The mTBI patients did not differ from HCs regarding

2 age, education, gender, and mean FD (Table 1).

3 Significant differences were revealed between patients with mTBI in acute stage and HCs in TMT-A (p

$4=0.023), \operatorname{DSC}(\mathrm{p}=0.021), \operatorname{RPCS}(\mathrm{p}<0.001)$, ISI $(\mathrm{p}<0.001)$, and PCL-C $(\mathrm{p}<0.001)($ Table $1, \mathrm{p} 1$

5 represent the p-value from statistical test between acute mTBI patients and HCs). And significant

6 differences in Forward DS $(p=0.037)$, Backward DS $(p=0.008)$, RPCS $(p=0.014)$, ISI $(p=0.009)$,

7 and PCL-C ( 1 < 0.001) were observed between patients with mTBI in chronic stage and HCs (Table 1,

8 p2 represent the p-value from statistical test between chronic mTBI patients and HCs). The detailed

9 demographic information and cognitive and clinical assessments of mTBI and HCs were demonstrated

10 in Table 1.

\section{WM-FNs}

12 The coefficient of Dice was adopted to evaluate the stability of WM-FNs number, the results 13 demonstrated the most stable (Dice's coefficient $>0.85$ ) with the largest number of WM-FNs was 11 14 (Fig. 2). The WM-FN is given a putative network name based on the anatomical location. The 15 WM-FNs can be named as: WM1 (IFOF network), WM2 (corona radiate network), WM3(anterior 16 temporal network), WM4 (orbito-frontal network), WM5 (pre/postcentral network), WM6 (superior 17 longitudinal fasciculus network), WM7 (ventral frontal network), WM8 (temporoparietal network), 18 WM9 (Rolandic network), WM10 (cerebellar network), WM11 (occipital network) (Fig. 2, Table 2).

19 Besides, based on the spatial distribution [24], we defined middle/superficial WM-FNs (WM-FN 2, 3,4 ,

$205,7,8,9,10,11$ ) and deep WM-FNs (WM-FN 1, 6) (Fig. 2, Table 2)

\section{Similarity between WM-FNs and DTI fiber tracts}

22 Four WM-FN $(2,5,9,10)$ of the 11 networks observed spatial correspondence with specific DTI

23 anatomical tracts (e.g., network 9 and superior longitudinal fasciculus;) (Fig. 3, Table 2). And the other

24 networks spatial corresponded to multiple DTI tracts (e.g., network 1 spatial corresponded to both

25 IFOF and inferior longitudinal fasciculus;) (Fig. 3, Table 2). 


\section{Functional connectivity}

\section{Functional connectivity and its association with cognitive deficits in the}

\section{3 acute stage}

4 Across subjects, middle/superficial WM-FNs demonstrated high correlation in their spontaneous

5 activity with their adjacent GM-FNs and some distributed GM-FNs, such as occipital network and

6 visual network $(\mathrm{r}=0.92)$, rolandic network and sensorimotor network $(\mathrm{r}=0.92)$, temporoparietal

7 network and default mode network ( $\mathrm{r}=0.87)$ (Fig. 4 (a), Table 2). Besides, a relatively weak

8 correlation $(\mathrm{r}<0.55)$ was revealed between the deep WM-FNs and GM-FNs (Fig. 4 (a)).

9 Across subjects, between adjacent WM-FNs, such as the superior longitudinal fasciculus network and 10 IFOF network, showed a strong correlation $(\mathrm{r}=0.74)$ (Fig. 4 (b), Table 2). Between distant WM-FNs, 11 such as cerebellar WM-FN and the other WM-FNs, showed relatively weak correlation $(\mathrm{r}<0.60)($ Fig. $124(\mathrm{~b}))$

13 Compared to HCs, patients with mTBI showed increased FC between the deep WM-FN and low-level 14 primary sensorimotor cortical GM-FNs. In detail, mTBI patients showed increased FC between IFOF 15 WM-FN and visual GM-FN, IFOF WM-FN and anterior cerebellar GM-FN, IFOF WM-FN and 16 sensorimotor GM-FN (Fig. 4 (c), Table 3).

17 Compared to HCs, patients with mTBI also showed increased FC between deep WM-FN and low-level 18 primary sensorimotor superficial WM-FN. In detail, patients with mTBI showed increased FC between 19 IFOF WM-FN and occipital WM-FN. Also, patients with mTBI showed increased FC between IFOF 20 WM-FN and pre/postcentral WM-FN (Fig. 4 (c), Table 3).

21 Taken together, the IFOF WM-FN is the most affected WM-FN, which showed increased connectivity

22 with low-level primary sensorimotor functional networks in acute mTBI patients.

23 The FC between IFOF WM-FN and anterior cerebellar GM-FN was positively correlated with TMT-A 24 score (Fig. 4 (d)). 


\section{Functional connectivity and its association with cognitive deficits in the}

\section{2 chronic stage}

3 Similar to the acute stage, the interaction between WM-FNs and GM-FNs in the chronic stage showed

4 a relatively high correlation between middle/superficial WM-FNs and adjacent GM-FNs, such as the

5 occipital network and visual network $(\mathrm{r}=0.92)$, rolandic network and sensorimotor network $(\mathrm{r}=0.92)$,

6 temporoparietal network and default mode network $(\mathrm{r}=0.87)$. The deep WM-FN and GM-FNs showed

7 a relatively weak correlation $(\mathrm{r}<0.55)($ Fig. $5(\mathrm{a}))$.

8 The interaction between pair of WM-FNs in the chronic stage was also similar to the acute stage with a

9 strong correlation between adjacent WM-FNs, such as the superior longitudinal fasciculus network and

10 IFOF network $(\mathrm{r}=0.75)$ (Fig. $5(\mathrm{~b}))$. A relatively weak correlation was presented between distant

11 WM-FNs, such as cerebellar WM-FN and the other WM-FNs ( $r<0.60)$ (Fig. 5 (b)).

12 The mTBI patients did not differ from HCs regarding FC between WM and GM-FNs (Fig. 5 (c)). Also,

13 mTBI patients and HCs did not differ regarding FC within WM-FNs (Fig. 5 (d)).

14 Besides, even the WM-FNs were constructed by the patients in chronic stage and HCs, the results 15 remain stable (see in supplementary material).

\section{Discussion}

17 The present study, for the first time, investigated the dynamic change of WM-FN in mTBI within a 18 longitudinal study. Eleven distinct WM-FNs were identified by clustering voxel-wise WM functional 19 connectivity. These networks are corresponded to either a specific or multiple anatomical DTI WM 20 tracts. The altered FC of WM-FN was observed in the acute stage but not in the chronic stage, 21 suggesting a possible compensatory mechanism in the chronic recovery stage. Importantly, the IFOF 22 WM-FN demonstrated enhanced FC both with the low-level GM primary sensorimotor networks, and 23 with the low-level WM primary sensorimotor networks in acute mTBI patients. These enhanced FC 24 may represent a compensatory or protective mechanism in response to cognitive deficits in acute mTBI.

25 The convergent damage of IFOF network highlighted the importance of IFOF WM-FN in our 
1 understanding of the pathophysiology mechanism of mTBI patients, which can further aid the

2 promotion of new treatment intervention that targeting IFOF network in the acute stage.

\section{The spatial similarity between WM-FNs and DTI fiber tracts}

4 In previous studies, the WM tracts integrity analysis of mTBI patients were observed mainly based on

5 structural MRI, such as DTI. The current study reported a novel observation of functional

6 communication of WM in mTBI patients. The constructed WM-FNs were corresponded to either a

7 specific or multiple anatomically DTI WM tracts. This is consistent with the previous study [27] to

8 support the notion that multiple DTI tracts collaborate with each other to guarantee the specific brain

9 function. The analysis of spatial interacting patterns between DTI fiber tracts and WM-FNs helps to

10 clarify which DTI fiber tract can be reflected by the WM-FN, especially by the deep WM networks.

11 For example, the identified IFOF functional network was mainly a spatial interaction with IFOF fiber

12 tract (Fig. 3, Table 2). Thus, the IFOF functional network was assumed to mainly reflect the function of

13 IFOF fiber tract. It should be noted that it is difficult to describe middle/superficial WM-FNs using DTI

14 fiber tract since they combine many small fibers with different orientations [45]. Therefore, the

15 function of middle/superficial WM-FNs is mainly reflected by their connectivity with cortical GM-FNs.

16 For instance, the occipital WM-FN showed strong connectivity with cortical visual GM-FN, so that the

17 occipital WM-FN was assumed to also reflect the visual function to some extent. In sum, and together

18 with previous study [46,21,27], these results provide further support that WM-FNs have corresponding

19 structural base.

\section{Functional connectivity in the acute stage}

21 Intriguingly, the IFOF WM-FN was found to be the most affected deep WM-FN in mTBI patients. The

22 IFOF WM-FN showed enhanced FC both with the low-level GM primary sensorimotor networks

23 (visual network, anterior cerebellar network, and sensorimotor network), and with the low-level WM

24 primary sensorimotor networks (occipital network and pre/postcentral network) in patients with mTBI

25 at the acute stage. Previous studies have reported the damaged structure property in IFOF in patients

26 with mTBI $[47,48]$. Here, for the first time, the present study showed that increased IFOF FC with 
1 low-level WM and GM primary sensorimotor networks in patients with mTBI. In general, the

2 increased positive FC reflects an increased integrative ability sub-serving similar goals [49]. Hence, the

3 increased IFOF FC may reflect a compensatory mechanism in mTBI. As a long-distance associative

4 tract, the IFOF plays a crucial role in connecting the occipital lobe, the parietal lobe, and the

5 poster-temporal cortex with the frontal lobe [13], which was particularly vulnerable to the mechanical

6 trauma of TBI [11]. Correspondingly, the IFOF is crucial in sending the processed visual information

7 from the occipital lobe to frontal lobe, where information was further translated into semantic

8 information [50]. The observed increased connectivity between IFOF FC with low-level WM and GM

9 primary sensorimotor networks may represent a compensatory effect in response to cognitive deficits.

10 These enhanced FCs may indicate mTBI patients employed higher cognitive effort in integrating

11 dynamic environmental sensory information and high-order cognitive function to coordinate their behaviors and thoughts to fit with contextual information. This interpretation is supported by previous

13 studies which demonstrated abnormal activation of the intrinsic rich-club network in mTBI patients [51]

14 and greater network strength in mTBI patients without post-concussive syndrome [52]. Both studies

15 suggested a potential compensatory or protective mechanism in mTBI patients. The current findings

16 highlighted the importance of the IFOF WM-FN in such a compensatory mechanism, in which IFOF

17 WM-FN may excessively contribute to integrating sensory information from GM and WM primary

18 sensory-related network with high-order cognitive information in acute mTBI patients. The most

19 affected damage in mTBI was located in IFOF network, which implies the crucial role of IFOF

20 network in our understanding of the pathophysiology mechanism of mTBI patients. This observation

21 can further aid the promotion of new treatment intervention targeting IFOF network in the acute stage.

\section{The association between altered WM-FNs and cognitive}

\section{3 assessments}

24 Besides, the FC between IFOF WM-FN and anterior cerebellar GM-FN was positively correlated with

25 TMT-A score in mTBI patients at acute stage. This correlation suggested a deficit of the information

26 processing speed in acute mTBI patients. Information processing speed is depend on large-scale and

27 long-distance neural network communications, which is among the earliest and most prominent 
1 cognitive manifestations in mTBI [53,54]. Thus, the deficit of information processing speed was

2 assumed to be caused by the damage of IFOF network in acute mTBI patients to some extent.

\section{Functional connectivity in the chronic stage}

4 Furthermore, mTBI patients at the chronic stage did not differ from HCs in FC within WM-FNs or

5 between GM-FN and WM-FN. A previous meta-analysis indicated that the damage effects for memory

6 and fluency were greatest in the acute stage (less than 3 months postinjury) and no residual impairment

7 by 3 months postinjury [55]. Besides, a recent large sample longitudinal neuroimaging study of TBI

8 revealed a 'U-shaped' curve in sub-acute, 1 year, and 5 years postinjury in the number of fractional

9 anisotropy abnormalities [56]. Furthermore, a previous study demonstrated a functional and structural

10 abnormal connectivity in early phase of mTBI, but a considerable compensation of functional and

11 structural connectivity subsequent to the acute phase [57]. Combining previous studies and the current

12 findings, it is evident that the compensatory mechanism of brain function contributed to the recovery of

13 cognition deficits in mTBI patients at the chronic stage.

\section{Limitation}

15 Notwithstanding its implication, limitations of the current study should be acknowledged and addressed.

16 Some researchers have speculated that the WM signals might have infiltrated from the GM for partial

17 volume effect. To avoid the influence of GM signals, the spatial smooth of WM and GM signals were

18 separately carried out within individual WM mask or GM mask to avoid the mixture of WM and GM

19 signals. Besides, only the data of mTBI patients in the acute stage and the chronic stage (6-12 months

20 postinjury) was collected, the data for longer follow-up, such as 5 years postinjury should be collected

21 as a follow-up. Based on a previous study [56], the significant abnormalities of FA in TBI were

22 observed at the sub-acute and 5 years postinjury, but not 1-year postinjury. Therefore, it is necessary to

23 follow patients for a longer period to explore the development of the observed deficits of WM-FNs. 


\section{Conclusion}

2 In summary, this study constructed WM-FNs based k-means clustering algorithm and revealed an

3 excessive interaction between deep WM-FNs and low-level primary sensorimotor networks, which can

4 be regarded as a compensatory mechanism of cognitive deficits in mTBI patients in the acute stage. Of

5 note, patients with mTBI did not differ from HCs at the chronic stage in FC within WM-FNs or

6 between GM and WM-FN, suggesting a possible compensatory mechanism of brain function

7 contributed to the recovery of cognition deficits in mTBI patients at the chronic stage. The convergent

8 damage of IFOF network highlighted its key role in our understanding of the pathophysiology

9 mechanism of mTBI patients and thus might be regarded as a biomarker in the acute stage and a

10 potential indicator of treatment effect.

\section{Funding}

12 This study was supported by the National Science Foundation of China [grant number 81771914], the

13 Humanities and Social Science Foundation of Ministry of Education of China [grant number

14 19YJC740100], and the Fundamental Research Funds for the Central Universities [grant number 15 RW180178].

\section{Conflicts of interest}

17 The authors declare that they have no conflict of interest. 


\section{Supplementary material}

\section{Detailed inclusion and exclusion criteria of mTBI patients}

3 In detail, patients with mTBI were selected with the following criteria: (a) Glasgow Coma Score of

4 13-15; (b) one or more of the following: confusion or disorientation, post-traumatic amnesia for less

5 than 24 hours, loss of consciousness for 30 minutes or less, and/or other transient neurological

6 abnormalities such as focal signs, seizure, and intracranial lesion not requiring surgery; (c) diagnosed

7 within 1 week after onset of mTBI. The exclusion criteria included: (a) a history of a previous brain

8 injury, neurological disease, long-term psychiatric history, or a history of concurrent substance or

9 alcohol abuse; (b) a structural abnormality in neuroimaging (CT and MRI); (c) intubation and/or skull

10 fracture, and administration of sedatives; (d) the manifestation of mTBI caused by medications, alcohol,

11 drugs for other injuries (such as systemic injuries, facial injuries, or intubation); (e) other problems

12 (such as psychological trauma, language impairment, or coexisting medical conditions); (f) caused by

13 penetrating craniocerebral injury.

14 Image acquisition

15 The rs-fMRI data was acquired using a gradient-recalled echo planar imaging (EPI) sequence, and the 16 scan parameters were as follows: repetition time $(\mathrm{TR})=2500 \mathrm{~ms}$, echo time $(\mathrm{TE})=30 \mathrm{~ms}$, slice 17 thickness $=3 \mathrm{~mm}$, flip angle $(\mathrm{FA})=90^{\circ}$, field of view $(\mathrm{FOV})=216 \mathrm{~mm} \times 216 \mathrm{~mm}$, matrix size $=64 \times$ 18 64. One hundred and eighty volumes were obtained. The scan parameters of High-resolution 19 T1-weighted 3D BRAVO sequence were as follows: $\mathrm{TR}=8.15 \mathrm{~ms}$, $\mathrm{TE}=3.17 \mathrm{~ms}$, slice thickness $=1$ $20 \mathrm{~mm}, \mathrm{FA}=9^{\circ}, \mathrm{FOV}=256 \mathrm{~mm} \times 256 \mathrm{~mm}$. The scan parameters of DTI were as follows: $\mathrm{TR}=8000 \mathrm{~ms}$, $21 \mathrm{TE}=68 \mathrm{~ms}, \mathrm{FA}=90^{\circ}$, slice thickness $=2 \mathrm{~mm}$, slices $=75$, matrix size $=128 \times 128$, two averages, FOV $22=256 \mathrm{~mm} \times 256 \mathrm{~mm}$, voxel size $=2 \mathrm{~mm} \times 2 \mathrm{~mm} \times 2 \mathrm{~mm}$. DTI $(\mathrm{b}=1000 \mathrm{~s} / \mathrm{mm} 2)$ used 30 diffusion 23 gradient orientations and the unweighted diffusion imaging $(b=0)$ repeated five times. During 24 scanning, all participants were instructed to relax, close their eyes, keep awake, and try not to think of 25 anything in particular.

\section{WM-FNs clustering of the chronic stage}

27 The WM-FNs clustering based on the data of patients in the chronic stage and HCs data were also 28 calculated to avoid the functional reconstruction of WM-FNs in the chronic stage. According to the 29 results of Dice's coefficient (Supp Fig. 1), we identified 12 WM-FNs (Supp Fig. 1). Compared with 30 HCs, there also was no significant difference between patients and healthy control in functional 
bioRxiv preprint doi: https://doi.org/10.1101/2020.09.25.313338: this version posted September 28. 2020. The copvriaht holder for this preprint (which was not certified by peer review) is the author/funder, who has granted bioRxiv a license to display the preprint in perpetuity. It is made available under aCC-BY-ND 4.0 International license.

1 connectivity between WM-FNs and GM functional connectivity, and no significant difference between

2 patients and healthy control in functional connectivity within WM-FNs in the chronic stage of mTBI

3 (Supp Fig. 2).

4 


\section{References}

2 1. Feigin VL, Theadom A, Barker-Collo S, Starkey NJ, McPherson K, Kahan M, Dowell A, Brown P,

3 Parag V, Kydd R, Jones K, Jones A, Ameratunga S, Group BS (2013) Incidence of traumatic brain

4 injury in New Zealand: a population-based study. Lancet Neurol 12 (1):53-64.

5 doi:10.1016/S1474-4422(12)70262-4

6 2. Maas AIR, Menon DK, Adelson PD, Andelic N, Bell MJ, Belli A, Bragge P, Brazinova A, Buki A,

7 Chesnut RM, Citerio G, Coburn M, Cooper DJ, Crowder AT, Czeiter E, Czosnyka M, Diaz-Arrastia R,

8 Dreier JP, Duhaime AC, Ercole A, van Essen TA, Feigin VL, Gao G, Giacino J, Gonzalez-Lara LE,

9 Gruen RL, Gupta D, Hartings JA, Hill S, Jiang JY, Ketharanathan N, Kompanje EJO, Lanyon L,

10 Laureys S, Lecky F, Levin H, Lingsma HF, Maegele M, Majdan M, Manley G, Marsteller J, Mascia L,

11 McFadyen C, Mondello S, Newcombe V, Palotie A, Parizel PM, Peul W, Piercy J, Polinder S,

12 Puybasset L, Rasmussen TE, Rossaint R, Smielewski P, Soderberg J, Stanworth SJ, Stein MB, von

13 Steinbuchel N, Stewart W, Steyerberg EW, Stocchetti N, Synnot A, Te Ao B, Tenovuo O, Theadom A,

14 Tibboel D, Videtta W, Wang KKW, Williams WH, Wilson L, Yaffe K, In TP, Investigators (2017)

15 Traumatic brain injury: integrated approaches to improve prevention, clinical care, and research.

16 Lancet Neurol 16 (12):987-1048. doi:10.1016/S1474-4422(17)30371-X

17 3. Robertson CS, Garcia R, Gaddam SS, Grill RJ, Cerami Hand C, Tian TS, Hannay HJ (2013)

18 Treatment of mild traumatic brain injury with an erythropoietin-mimetic peptide. J Neurotrauma 30

19 (9):765-774. doi:10.1089/neu.2012.2431

20 4. Bazarian JJ, Zhong J, Blyth B, Zhu T, Kavcic V, Peterson D (2007) Diffusion tensor imaging detects

21 clinically important axonal damage after mild traumatic brain injury: a pilot study. J Neurotrauma 24

22 (9):1447-1459. doi:10.1089/neu.2007.0241

23 5. Kirov, II, Tal A, Babb JS, Reaume J, Bushnik T, Ashman TA, Flanagan S, Grossman RI, Gonen O

24 (2013) Proton MR spectroscopy correlates diffuse axonal abnormalities with post-concussive

25 symptoms in mild traumatic brain injury. J Neurotrauma 30 (13):1200-1204.

26 doi:10.1089/neu.2012.2696

27 6. Gennarelli TA, Thibault LE, Adams JH, Graham DI, Thompson CJ, Marcincin RP (1982) Diffuse

28 axonal injury and traumatic coma in the primate. Ann Neurol 12 (6):564-574.

29 doi:10.1002/ana.410120611

30 7. Johnson VE, Stewart W, Smith DH (2013) Axonal pathology in traumatic brain injury. Exp Neurol 31 246:35-43. doi:10.1016/j.expneurol.2012.01.013

32 8. Smith DH, Hicks R, Povlishock JT (2013) Therapy development for diffuse axonal injury. J 33 Neurotrauma 30 (5):307-323. doi:10.1089/neu.2012.2825

34 9. Sharp DJ, Scott G, Leech R (2014) Network dysfunction after traumatic brain injury. Nat Rev 35 Neurol 10 (3):156-166. doi:10.1038/nrneurol.2014.15

36 10. Bonnelle V, Ham TE, Leech R, Kinnunen KM, Mehta MA, Greenwood RJ, Sharp DJ (2012)

37 Salience network integrity predicts default mode network function after traumatic brain injury. Proc

38 Natl Acad Sci U S A 109 (12):4690-4695. doi:10.1073/pnas.1113455109

39 11. Adams JH, Graham DI, Gennarelli TA, Maxwell WL (1991) Diffuse axonal injury in non-missile

40 head injury. J Neurol Neurosurg Psychiatry 54 (6):481-483. doi:10.1136/jnnp.54.6.481 


\section{4}

12. Kinnunen KM, Greenwood R, Powell JH, Leech R, Hawkins PC, Bonnelle V, Patel MC, Counsell SJ, Sharp DJ (2011) White matter damage and cognitive impairment after traumatic brain injury. Brain 134 (Pt 2):449-463. doi:10.1093/brain/awq347

13. Forkel SJ, Thiebaut de Schotten M, Kawadler JM, Dell'Acqua F, Danek A, Catani M (2014) The anatomy of fronto-occipital connections from early blunt dissections to contemporary tractography. Cortex 56:73-84. doi:10.1016/j.cortex.2012.09.005

14. Yin B, Li DD, Huang H, Gu CH, Bai GH, Hu LX, Zhuang JF, Zhang M (2019) Longitudinal Changes in Diffusion Tensor Imaging Following Mild Traumatic Brain Injury and Correlation With Outcome. Front Neural Circuits 13:28. doi:10.3389/fncir.2019.00028

15. Clark AL, Delano-Wood L, Sorg SF, Werhane ML, Hanson KL, Schiehser DM (2017) Cognitive fatigue is associated with reduced anterior internal capsule integrity in veterans with history of mild to moderate traumatic brain injury. Brain Imaging Behav 11 (5):1548-1554. doi:10.1007/s11682-016-9594-6

16. San Martin Molina I, Salo RA, Abdollahzadeh A, Tohka J, Grohn O, Sierra A (2020) In Vivo Diffusion Tensor Imaging in Acute and Subacute Phases of Mild Traumatic Brain Injury in Rats. eNeuro 7 (3). doi:10.1523/ENEURO.0476-19.2020

17. Mesulam MM (1998) From sensation to cognition. Brain 121 ( Pt 6):1013-1052. doi:10.1093/brain/121.6.1013

18. Dong D, Luo C, Guell X, Wang Y, He H, Duan M, Eickhoff SB, Yao D (2020) Compression of Cerebellar Functional Gradients in Schizophrenia. Schizophr Bull. doi:10.1093/schbul/sbaa016 19. Jia X, Xie Y, Dong D, Pei H, Jiang S, Ma S, Huang Y, Zhang X, Wang Y, Zhu Q, Zhang Y, Yao D, Yu L, Luo C (2020) Reconfiguration of dynamic large-scale brain network functional connectivity in generalized tonic-clonic seizures. Hum Brain Mapp 41 (1):67-79. doi:10.1002/hbm.24787

20. Ding Z, Huang Y, Bailey SK, Gao Y, Cutting LE, Rogers BP, Newton AT, Gore JC (2018) Detection of synchronous brain activity in white matter tracts at rest and under functional loading. Proc Natl Acad Sci U S A 115 (3):595-600. doi:10.1073/pnas.1711567115 21. Ding Z, Xu R, Bailey SK, Wu TL, Morgan VL, Cutting LE, Anderson AW, Gore JC (2016) Visualizing functional pathways in the human brain using correlation tensors and magnetic resonance imaging. Magn Reson Imaging 34 (1):8-17. doi:10.1016/j.mri.2015.10.003

22. Wu TL, Wang F, Anderson AW, Chen LM, Ding Z, Gore JC (2016) Effects of anesthesia on resting state BOLD signals in white matter of non-human primates. Magn Reson Imaging 34 (9):1235-1241. doi:10.1016/j.mri.2016.07.001

23. Fabri M, Polonara G, Mascioli G, Salvolini U, Manzoni T (2011) Topographical organization of human corpus callosum: an fMRI mapping study. Brain Res 1370:99-111. doi:10.1016/j.brainres.2010.11.039

24. Gawryluk JR, Mazerolle EL, Brewer KD, Beyea SD, D'Arcy RC (2011) Investigation of fMRI activation in the internal capsule. BMC Neurosci 12:56. doi:10.1186/1471-2202-12-56

25. Wu X, Yang Z, Bailey SK, Zhou J, Cutting LE, Gore JC, Ding Z (2017) Functional connectivity 9 and activity of white matter in somatosensory pathways under tactile stimulations. Neuroimage 40 152:371-380. doi:10.1016/j.neuroimage.2017.02.074 
26. Marussich L, Lu KH, Wen H, Liu Z (2017) Mapping white-matter functional organization at rest and during naturalistic visual perception. Neuroimage 146:1128-1141. doi:10.1016/j.neuroimage.2016.10.005

27. Peer M, Nitzan M, Bick AS, Levin N, Arzy S (2017) Evidence for Functional Networks within the Human Brain's White Matter. J Neurosci 37 (27):6394-6407. doi:10.1523/JNEUROSCI.3872-16.2017 28. Ji GJ, Ren C, Li Y, Sun J, Liu T, Gao Y, Xue D, Shen L, Cheng W, Zhu C, Tian Y, Hu P, Chen X, Wang K (2019) Regional and network properties of white matter function in Parkinson's disease. Hum Brain Mapp 40 (4):1253-1263. doi:10.1002/hbm.24444

29. Jiang Y, Luo C, Li X, Li Y, Yang H, Li J, Chang X, Li H, Yang H, Wang J, Duan M, Yao D (2019) White-matter functional networks changes in patients with schizophrenia. Neuroimage 190:172-181. doi:10.1016/j.neuroimage.2018.04.018

30. Jiang Y, Song L, Li X, Zhang Y, Chen Y, Jiang S, Hou C, Yao D, Wang X, Luo C (2019) Dysfunctional white-matter networks in medicated and unmedicated benign epilepsy with centrotemporal spikes. Hum Brain Mapp 40 (10):3113-3124. doi:10.1002/hbm.24584

31. Holm L, Cassidy JD, Carroll LJ, Borg J (2005) Summary of the WHO collaborating centre for neurotrauma task force on mild traumatic brain injury. J Rehabil Med 37 (3):137-141. doi:10.1080/16501970510027321

32. Oldfield RC (1971) The assessment and analysis of handedness: the Edinburgh inventory. Neuropsychologia 9 (1):97-113. doi:10.1016/0028-3932(71)90067-4

33. Arnett JA, Labovitz SS (1995) Effect of Physical Layout in Performance of the Trail Making Test. Psychol Assessment 7 (2):220-221

34. Harman-Smith YE, Mathias JL, Bowden SC, Rosenfeld JV, Bigler ED (2013) Wechsler Adult Intelligence Scale-Third Edition profiles and their relationship to self-reported outcome following traumatic brain injury. J Clin Exp Neuropsyc 35 (8):785-798. doi:10.1080/13803395.2013.824554

35. Joy S, Kaplan E, Fein D (2004) Speed and memory in the WAIS-III Digit Symbol--Coding subtest across the adult lifespan. Arch Clin Neuropsychol 19 (6):759-767. doi:10.1016/j.acn.2003.09.009

36. King NS, Crawford S, Wenden FJ, Moss NE, Wade DT (1995) The Rivermead Post Concussion Symptoms Questionnaire: a measure of symptoms commonly experienced after head injury and its reliability. J Neurol 242 (9):587-592. doi:10.1007/bf00868811

37. Sadeghniiat-Haghighi K, Montazeri A, Khajeh-Mehrizi A, Nedjat S, Aminian O (2014) The Insomnia Severity Index: cross-cultural adaptation and psychometric evaluation of a Persian version. Qual Life Res 23 (2):533-537. doi:10.1007/s11136-013-0489-3

38. Weathers FW, Litz BT, Herman D, Huska J, Keane T (1994) The PTSD checklist-civilian version (PCL-C). Boston, MA: National Center for PTSD 10

39. Yan CG, Wang XD, Zuo XN, Zang YF (2016) DPABI: Data Processing \& Analysis for (Resting-State) Brain Imaging. Neuroinformatics 14 (3):339-351. doi:10.1007/s12021-016-9299-4 40. Jenkinson M, Beckmann CF, Behrens TE, Woolrich MW, Smith SM (2012) Fsl. Neuroimage 62 (2):782-790. doi:10.1016/j.neuroimage.2011.09.015

41. Lorio S, Fresard S, Adaszewski S, Kherif F, Chowdhury R, Frackowiak RS, Ashburner J, Helms G, Weiskopf N, Lutti A, Draganski B (2016) New tissue priors for improved automated classification of subcortical brain structures on MRI. Neuroimage 130:157-166. doi:10.1016/j.neuroimage.2016.01.062 
42. Desikan RS, Segonne F, Fischl B, Quinn BT, Dickerson BC, Blacker D, Buckner RL, Dale AM, Maguire RP, Hyman BT, Albert MS, Killiany RJ (2006) An automated labeling system for subdividing the human cerebral cortex on MRI scans into gyral based regions of interest. Neuroimage 31 (3):968-980. doi:10.1016/j.neuroimage.2006.01.021

43. Craddock RC, James GA, Holtzheimer PE, 3rd, Hu XP, Mayberg HS (2012) A whole brain fMRI atlas generated via spatially constrained spectral clustering. Hum Brain Mapp 33 (8):1914-1928. doi:10.1002/hbm.21333

44. Power JD, Cohen AL, Nelson SM, Wig GS, Barnes KA, Church JA, Vogel AC, Laumann TO, Miezin FM, Schlaggar BL, Petersen SE (2011) Functional network organization of the human brain. Neuron 72 (4):665-678. doi:10.1016/j.neuron.2011.09.006

45. Oishi K, Zilles K, Amunts K, Faria A, Jiang H, Li X, Akhter K, Hua K, Woods R, Toga AW, Pike GB, Rosa-Neto P, Evans A, Zhang J, Huang H, Miller MI, van Zijl PC, Mazziotta J, Mori S (2008) Human brain white matter atlas: identification and assignment of common anatomical structures in superficial white matter. Neuroimage 43 (3):447-457. doi:10.1016/j.neuroimage.2008.07.009

46. Ding Z, Newton AT, Xu R, Anderson AW, Morgan VL, Gore JC (2013) Spatio-temporal correlation tensors reveal functional structure in human brain. PLoS One 8 (12):e82107. doi:10.1371/journal.pone.0082107

47. Kim E, Seo HG, Lee HH, Lee SH, Choi SH, Cho WS, Wagner AK, Oh BM (2019) Altered White Matter Integrity after Mild to Moderate Traumatic Brain Injury. J Clin Med 8 (9). doi:10.3390/jcm8091318

48. Niogi SN, Mukherjee P, Ghajar J, Johnson CE, Kolster R, Lee H, Suh M, Zimmerman RD, Manley GT, McCandliss BD (2008) Structural dissociation of attentional control and memory in adults with and without mild traumatic brain injury. Brain 131 (Pt 12):3209-3221. doi:10.1093/brain/awn247

49. Fox MD, Snyder AZ, Vincent JL, Corbetta M, Van Essen DC, Raichle ME (2005) The human brain is intrinsically organized into dynamic, anticorrelated functional networks. Proc Natl Acad Sci U S A 102 (27):9673-9678. doi:10.1073/pnas.0504136102

50. Martino J, Brogna C, Robles SG, Vergani F, Duffau H (2010) Anatomic dissection of the inferior fronto-occipital fasciculus revisited in the lights of brain stimulation data. Cortex 46 (5):691-699. doi:10.1016/j.cortex.2009.07.015

51. Antonakakis M, Dimitriadis SI, Zervakis M, Papanicolaou AC, Zouridakis G (2017) Reconfiguration of dominant coupling modes in mild traumatic brain injury mediated by delta-band activity: A resting state MEG study. Neuroscience 356:275-286. doi:10.1016/j.neuroscience.2017.05.032

52. Sours C, Chen H, Roys S, Zhuo J, Varshney A, Gullapalli RP (2015) Investigation of Multiple Frequency Ranges Using Discrete Wavelet Decomposition of Resting-State Functional Connectivity in Mild Traumatic Brain Injury Patients. Brain Connect 5 (7):442-450. doi:10.1089/brain.2014.0333

53. Bartzokis G, Lu PH, Tingus K, Mendez MF, Richard A, Peters DG, Oluwadara B, Barrall KA, Finn JP, Villablanca P, Thompson PM, Mintz J (2010) Lifespan trajectory of myelin integrity and maximum motor speed. Neurobiol Aging 31 (9):1554-1562. doi:10.1016/j.neurobiolaging.2008.08.015

54. Bai L, Bai G, Wang S, Yang X, Gan S, Jia X, Yin B, Yan Z (2020) Strategic white matter injury 1 associated with long-term information processing speed deficits in mild traumatic brain injury. Hum 2 Brain Mapp. doi:10.1002/hbm.25135 
1 55. Belanger HG, Curtiss G, Demery JA, Lebowitz BK, Vanderploeg RD (2005) Factors moderating

2 neuropsychological outcomes following mild traumatic brain injury: a meta-analysis. J Int

3 Neuropsychol Soc 11 (3):215-227. doi:10.1017/S1355617705050277

4 56. Mac Donald CL, Barber J, Andre J, Panks C, Zalewski K, Temkin N (2019) Longitudinal

5 neuroimaging following combat concussion: sub-acute, 1 year and 5 years post-injury. Brain Commun

61 (1):fcz031. doi:10.1093/braincomms/fcz031

7 57. Dall'Acqua P, Johannes S, Mica L, Simmen HP, Glaab R, Fandino J, Schwendinger M, Meier C,

8 Ulbrich EJ, Muller A, Baetschmann H, Jancke L, Hanggi J (2017) Functional and Structural Network

9 Recovery after Mild Traumatic Brain Injury: A 1-Year Longitudinal Study. Front Hum Neurosci

10 11:280. doi:10.3389/fnhum.2017.00280

11

12 
bioRxiv preprint doi: https://doi.org/10.1101/2020.09.25.313338; this version posted September 28, 2020. The copyright holder for this preprint (which was not certified by peer review) is the author/funder, who has granted bioRxiv a license to display the preprint in perpetuity. It is made available under aCC-BY-ND 4.0 International license.

1
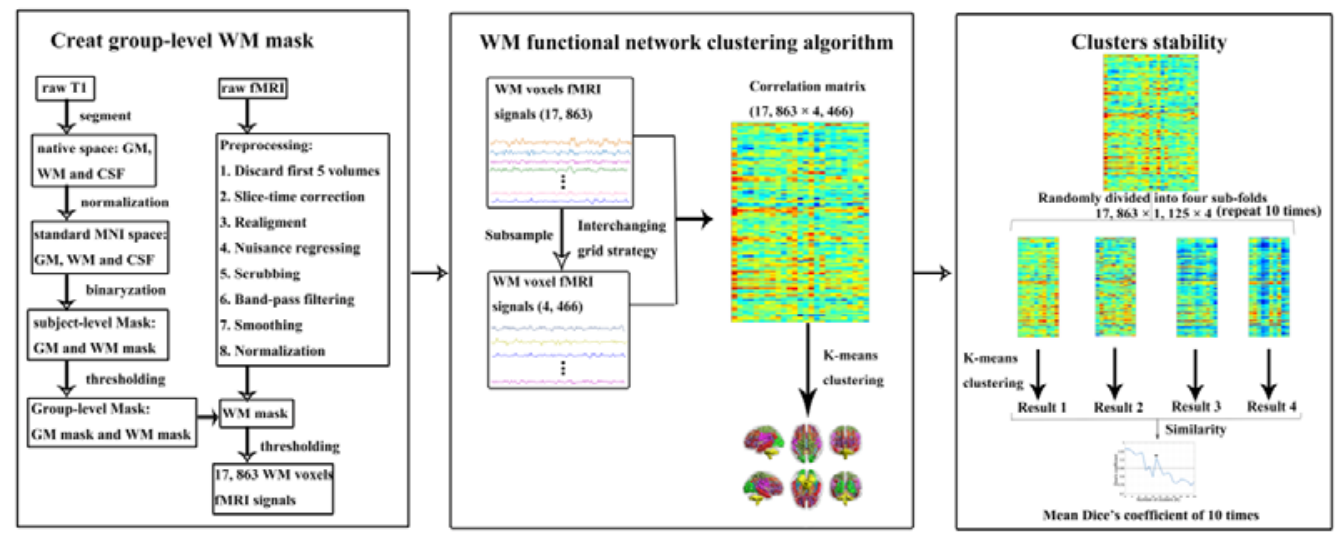

2 Fig. 1 Method overview of white matter functional networks clustering analysis

3 
bioRxiv preprint doi: https://doi.org/10.1101/2020.09.25.313338; this version posted September 28, 2020. The copyright holder for this preprint (which was not certified by peer review) is the author/funder, who has granted bioRxiv a license to display the preprint in perpetuity. It is made available under aCC-BY-ND 4.0 International license.

1
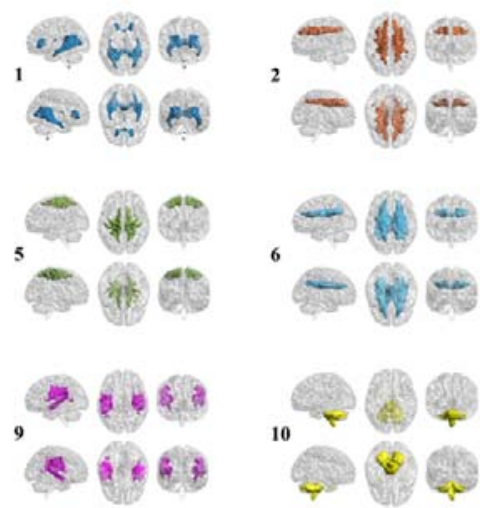

3
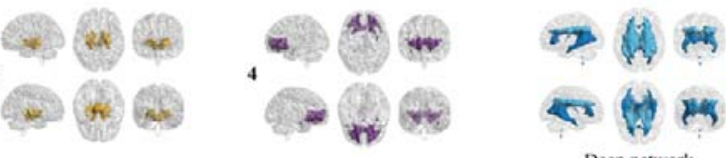

Deep network
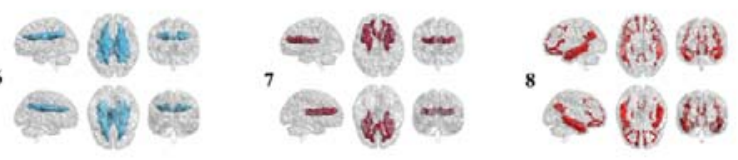

10
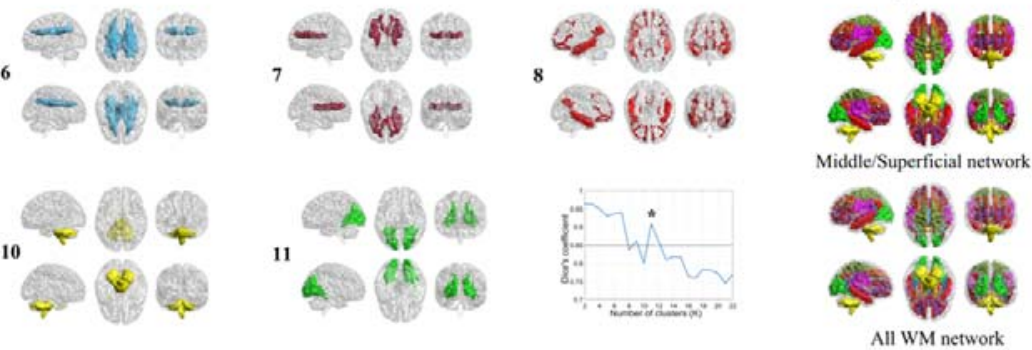

2 Fig. 2 Clusters stability, white matter functional networks (WM-FNs) of most suitable clusters $(\mathrm{k}=11)$,

3 and middle/superficial and deep WM-FNs

4 
bioRxiv preprint doi: https://doi.org/10.1101/2020.09.25.313338; this version posted September 28, 2020. The copyright holder for this preprint (which was not certified by peer review) is the author/funder, who has granted bioRxiv a license to display the preprint in perpetuity. It is made available under aCC-BY-ND 4.0 International license.

A.

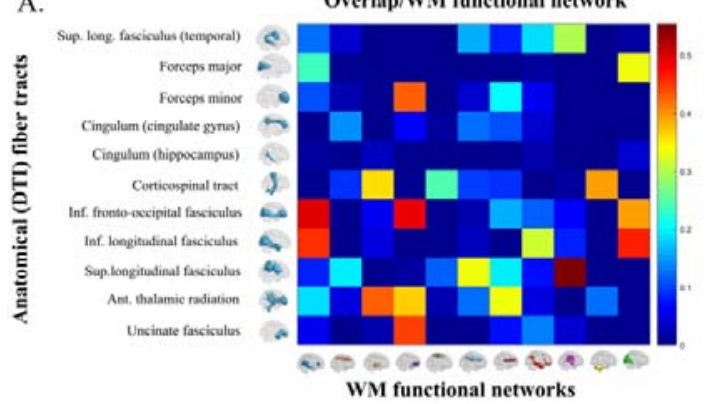

B.

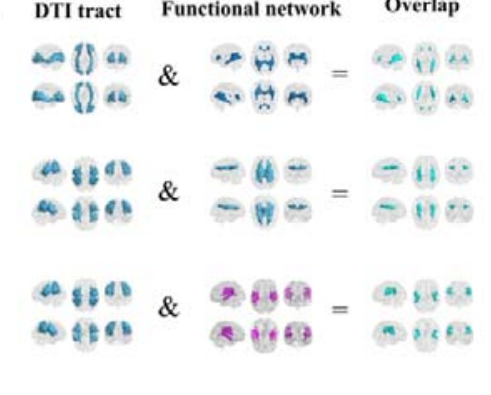

2 Fig. 3 The spatial similarity between the white matter functional networks (WM-FNs) and diffusion

3 tensor imaging (DTI) fiber tracts. (a) the ratio between the voxel number of overlapping and the voxel

4 number of WM-FN. (b) Examples about the spatial interaction between the WM-FN and DTI fiber tract 
A. FC between WM and GM network

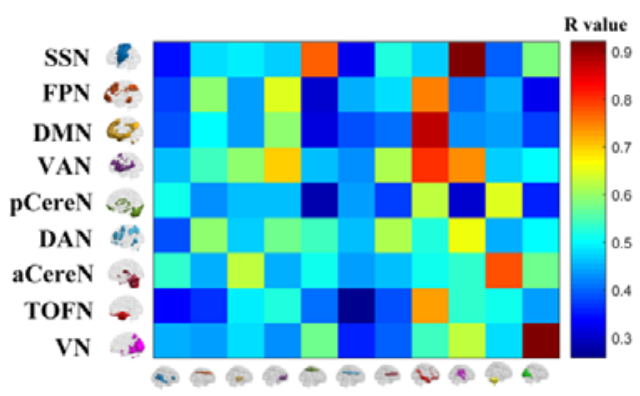

B. FC within WM networks

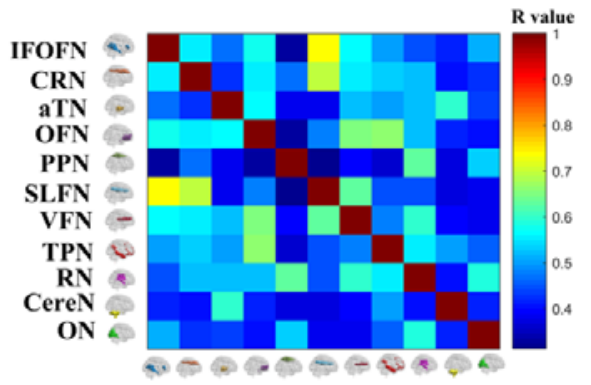

C. Group difference about FC

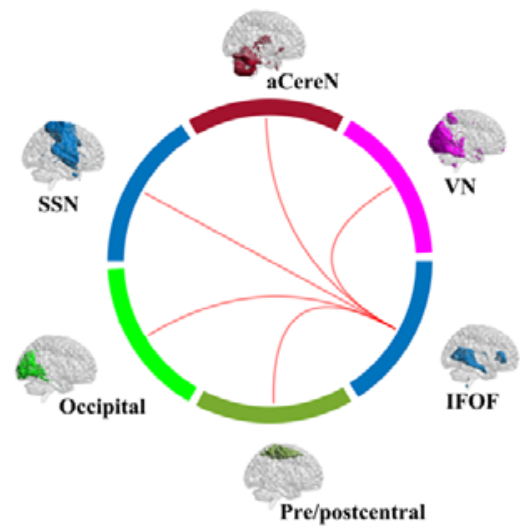

D. Correlations between altered WM network and cognition assessments

1

2 Fig. 4 Functional connectivity (FC) of white matter functional networks (WM-FNs) in the acute stage

3 mild traumatic brain injury (mTBI) patients and healthy controls (HCs). (a) Average FC between the

4 WM-FNs and grey matter functional networks (GM-FNs). (b) Average FC within WM-FNs. (c) Group

5 differences between patients and HCs in FC within WM-FNs or between WM-FN and GM-FN. The

6 red connections represented significantly increased FC in mTBI patients when compared with HCs

7 (FDR corrected, $\mathrm{p}<0.05$ ). (d) Correlations between abnormal FC and cognition assessments 
A. FC between WM and GM network

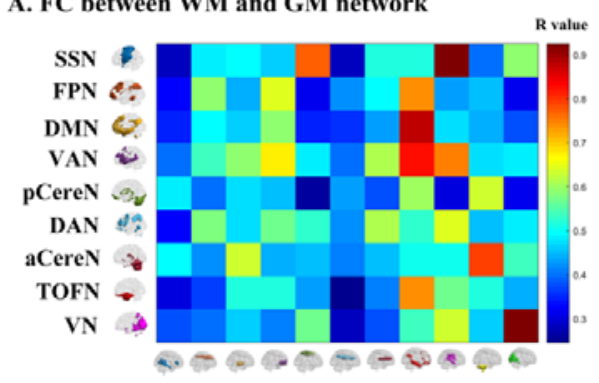

B. FC within WM networks

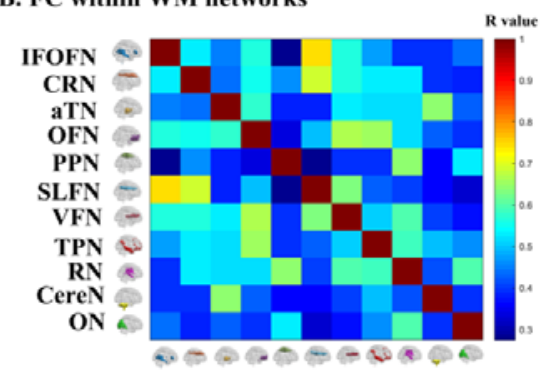

C. Group difference about FC between WM and GM network
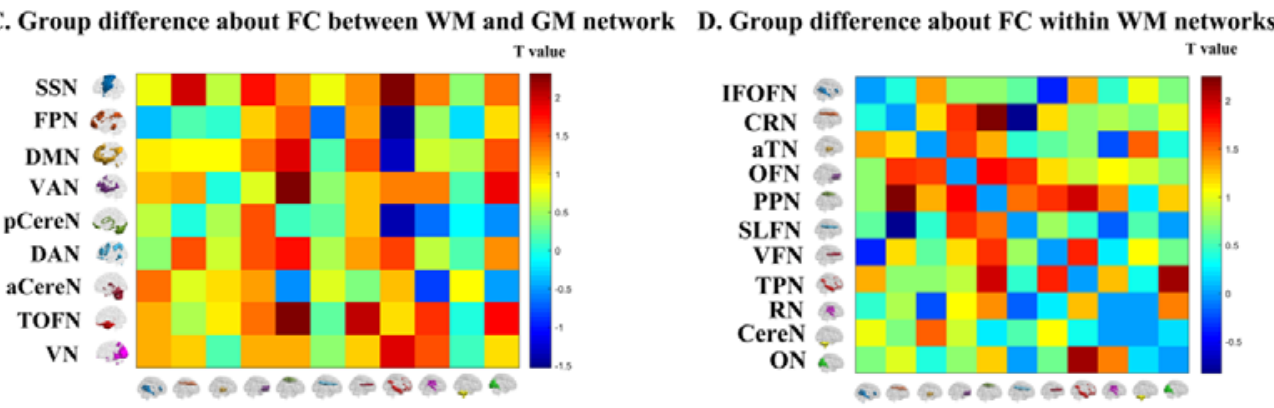

2 Fig. 5 Functional connectivity (FC) of white matter functional networks (WM-FNs) in the chronic

3 stage mild traumatic brain injury (mTBI) patients and healthy controls (HCs). (a) Average FC between

4 the WM-FNs and grey matter functional networks (GM-FNs). (b) Average FC within WM-FNs. (c)

5 Group differences of FC within distinct WM-FNs between mTBI patients and HCs. (d) Group

6 differences of FC between WM-FNs and GM-FNs between mTBI patients and HCs. The color bar in (a)

7 and (b) shows the Pearson's correlation coefficient (R value). The color bar in (c) and (d) shows the T

8 value from the two-sample t-test 
bioRxiv preprint doi: https://doi.org/10.1101/2020.09.25.313338; this version posted September 28,2020 . The copyright holder for this preprint (which was not certified by peer review) is the author/funder, who has granted bioRxiv a license to display the preprint in perpetuity. It is made available under aCC-BY-ND 4.0 International license.

1

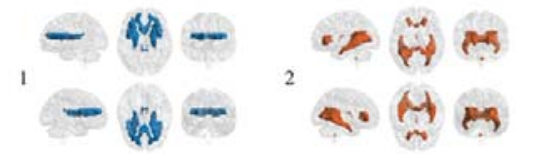

3
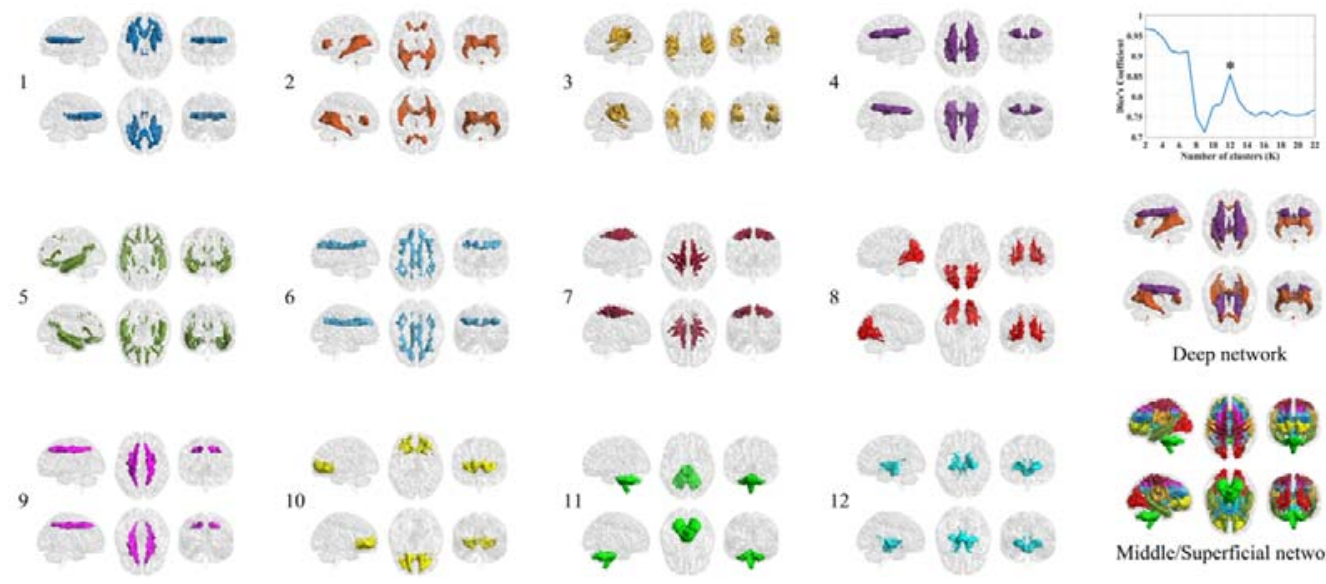

12
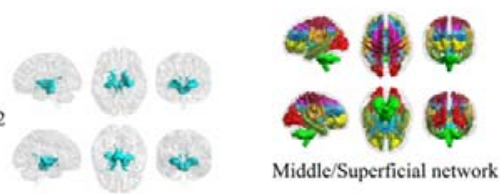

2 Supp Fig. 1 White matter functional networks identified by patients in the chronic stage and healthy

3 control

4 
A. FC within WM networks

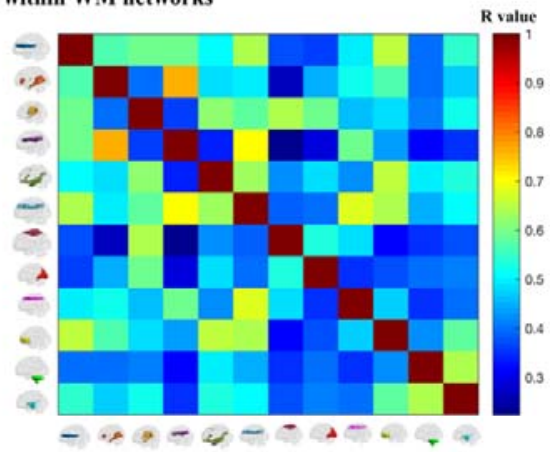

C. FC between WM and GM networks

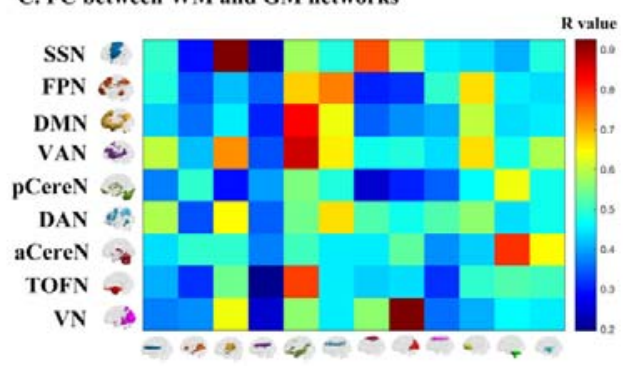

B. Group difference about FC within WM networks

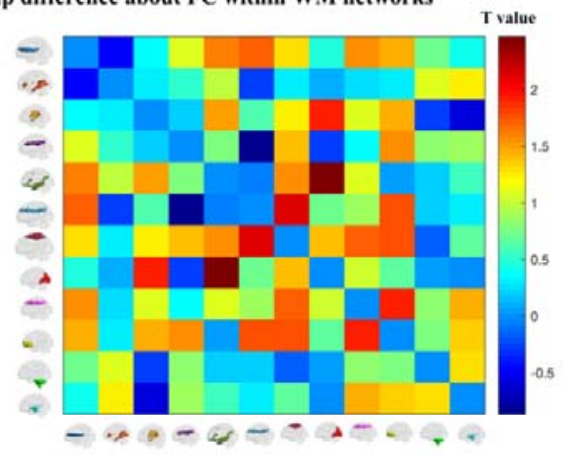

D. Group difference about FC between WM and GM networks

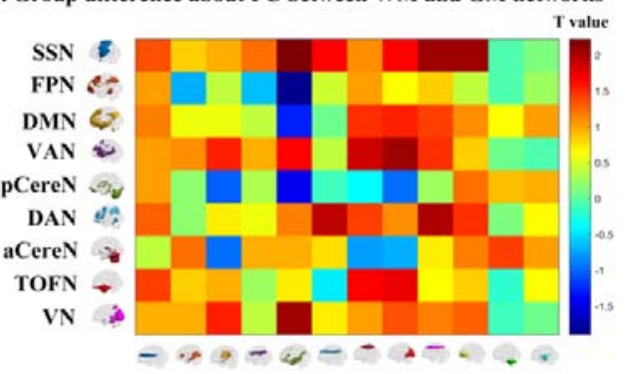

2 Supp Fig. 2 Functional connectivity (FC) of white matter functional networks (WM-FNs) in chronic

3 stage mild traumatic brain injury (mTBI) patients and healthy controls (HCs). (a) Average FC within

4 WM-FNs. (b) Group differences between mTBI patients and HCs in FC within WM-FNs (c) Average

5 FC between the WM-FNs and grey matter functional networks (GM-FNs). (d) Group differences

6 between mTBI patients and HCs in FC between WM-FNs and GM-FNs. The color bar in (a) and (c)

7 shows the Pearson's correlation coefficient (R value). The color bar in (b) and (d) shows the T value

8 from the two-sample t-test 
1 Table 1 Demographic characteristics of the mTBI patients and HCs

\begin{tabular}{llllll}
\hline characteristics & $\begin{array}{l}\mathrm{mTBI}(\mathrm{n}=97) \\
\text { (acute stage) }\end{array}$ & $\begin{array}{l}\mathrm{mTBI}(\mathrm{n}=56) \\
\text { (chronic stage) }\end{array}$ & HCs $(\mathrm{n}=43)$ & $\mathrm{p} 1$ & $\mathrm{p} 2$ \\
\hline Age (years) & $38.99 \pm 13.76$ & $35.32 \pm 15.32$ & $39.93 \pm 13.09$ & 0.454 & 0.064 \\
Gender (male:female) & $48: 49$ & $33: 23$ & $20: 23$ & 0.745 & 0.220 \\
Education (years) & $7.95 \pm 3.99$ & $8.54 \pm 3.77$ & $8.77 \pm 5.50$ & 0.598 & 0.804 \\
Mean FD & $0.11 \pm 0.10$ & $0.11 \pm 0.07$ & $0.11 \pm 0.06$ & 0.930 & 0.801 \\
Cognitive and Clinical assessments & $73.07 \pm 50.55$ & $44.86 \pm 35.88$ & $52.30 \pm 29.97$ & 0.023 & 0.109 \\
TMT-A & $31.99 \pm 16.30$ & $44.54 \pm 15.34$ & $39.63 \pm 17.72$ & 0.021 & 0.283 \\
DSC & $7.75 \pm 1.56$ & $8.57 \pm 1.59$ & $7.81 \pm 1.60$ & 0.835 & 0.037 \\
Forward DS & $3.80 \pm 1.47$ & $4.79 \pm 1.60$ & $3.95 \pm 1.45$ & 0.564 & 0.008 \\
Backward DS & $16.43 \pm 5.47$ & $18.86 \pm 6.19$ & $17.28 \pm 6.02$ & 0.389 & 0.147 \\
VF & $11 \pm 8.13$ & $5 \pm 5.30$ & $2.49 \pm 2.88$ & $<0.001$ & 0.014 \\
RPCS & $7.08 \pm 5.79$ & $3.39 \pm 4.53$ & $1.44 \pm 2.64$ & $<0.001$ & 0.009 \\
ISI & $25.12 \pm 6.18$ & $21.66 \pm 5.69$ & $17 \pm 0$ & $<0.001$ & $<0.001$ \\
PCL-C & & & & & \\
\hline
\end{tabular}

2 Abbreviations: mTBI, mild traumatic brain injury; HCs, healthy controls; TMT-A, trail making test A;

3 DSC, digit symbol coding; DS, digit span; VF, verbal fluency; RPCS, rivermead post-concussion

4 symptom questionnaire; ISI, insomnia severity index; PCL-C, posttraumatic stress disorder

5 checklist-civilian version.

$6 \mathrm{p} 1$ represents the p-values from statistical test between mTBI in acute stage and HCs.

$7 \mathrm{p} 2$ represents the p-values from statistical test between mTBI in chronic stage and HCs. 
1 Table 2 Overlap of WM-FNs with white matter fiber tracts, correlation with GM-FNs and WM-FNs

\begin{tabular}{|c|c|c|c|c|c|}
\hline Number & $\begin{array}{l}\text { Putative WM-FNs } \\
\text { designation }\end{array}$ & Layer & $\begin{array}{l}\text { Overlap/WM-FNs } \\
(>15 \%)\end{array}$ & $\begin{array}{l}\text { Correlation } \\
\text { with GM-FNs } \\
(r>0.7) \\
\end{array}$ & $\begin{array}{l}\text { Correlation with WM } \\
-\mathrm{FNs}(\mathrm{r}>0.7)\end{array}$ \\
\hline \multirow[t]{4}{*}{1} & \multirow[t]{4}{*}{ IFOF network } & \multirow[t]{4}{*}{ Deep } & IFOF $(50.23 \%)$ & - & SLF network (0.74) \\
\hline & & & $\operatorname{ILF}(45.72 \%)$ & - & - \\
\hline & & & Forceps major (23.98\%) & - & - \\
\hline & & & $\operatorname{ATR}(18.21 \%)$ & - & - \\
\hline 2 & Corona radiate network & Middle/Superficial & SLF $(19.86 \%)$ & - & - \\
\hline \multirow[t]{2}{*}{3} & \multirow[t]{2}{*}{ Anterior temporal network } & \multirow[t]{2}{*}{ Middle/Superficial } & $\operatorname{ATR}(42.61 \%)$ & - & - \\
\hline & & & Corticospinal tract $(35.81 \%)$ & - & - \\
\hline \multirow[t]{4}{*}{4} & \multirow[t]{4}{*}{ Orbitofrontal network } & \multirow[t]{4}{*}{ Middle/Superficial } & IFOF $(48.82 \%)$ & - & - \\
\hline & & & Uncinate fasciculus ( $44.63 \%$ ) & - & - \\
\hline & & & Forceps minor $(43.06 \%)$ & - & - \\
\hline & & & $\operatorname{ATR}(36.71 \%)$ & - & - \\
\hline 5 & Pre/postcentral network & Middle/Superficial & Corticospinal tract $(24.74 \%)$ & SSN (0.77) & - \\
\hline \multirow[t]{2}{*}{6} & \multirow[t]{2}{*}{ SLF network } & \multirow[t]{2}{*}{ Deep } & SLF $(32.96 \%)$ & - & IFOF network (0.74) \\
\hline & & & SLF (temporal) (16.35\%) & - & - \\
\hline \multirow[t]{4}{*}{7} & \multirow[t]{4}{*}{ Ventral frontal network } & \multirow[t]{4}{*}{ Middle/Superficial } & $\operatorname{ATR}(33.27 \%)$ & - & - \\
\hline & & & Forceps minor $(20.53 \%)$ & - & - \\
\hline & & & $\operatorname{SLF}(19.81 \%)$ & - & - \\
\hline & & & IFOF $(15.99 \%)$ & - & - \\
\hline \multirow[t]{4}{*}{8} & \multirow[t]{4}{*}{ Temporoparietal network } & \multirow[t]{4}{*}{ Middle/Superficial } & $\operatorname{ILF}(31.89 \%)$ & $\mathrm{DMN}(0.87)$ & - \\
\hline & & & SLF (temporal) (18.68\%) & VAN $(0.80)$ & - \\
\hline & & & - & FPN $(0.75)$ & - \\
\hline & & & - & TOFN $(0.73)$ & - \\
\hline \multirow[t]{2}{*}{9} & \multirow[t]{2}{*}{ Rolandic network } & \multirow[t]{2}{*}{ Middle/Superficial } & SLF $(55.44 \%)$ & $\mathrm{SSN}(0.92)$ & - \\
\hline & & & - & VAN $(0.73)$ & - \\
\hline 10 & Cerebellar network & Middle/Superficial & Corticospinal tract $(39.31 \%)$ & aCereN (0.78) & - \\
\hline \multirow[t]{3}{*}{11} & \multirow[t]{3}{*}{ Occipital network } & \multirow[t]{3}{*}{ Middle/Superficial } & $\operatorname{ILF}(46.76 \%)$ & $\mathrm{VN}(0.92)$ & - \\
\hline & & & IFOF $(39.44 \%)$ & - & - \\
\hline & & & Forceps major $(32.97 \%)$ & - & - \\
\hline
\end{tabular}

2 Abbreviations: WM-FNs, white matter functional networks; GM-FNs, grey matter functional networks;

3 IFOF, inferior fronto-occipital fasciculus; ILF, inferior longitudinal fasciculus; ATR, anterior thalamic

4 radiation; SLF, superior longitudinal fasciculus; SSN, sensorimotor network; DMN, default mode

5 network; VAN, ventral attention network; FPN, frontoparietal network; TOFN, temporal-orbitofrontal

6 network; aCereN, anterior cerebellar network; VN, visual network; 
1 Table 3 Group differences between mTBI patients in the acute stage and HCs in FC within WM-FNs

2 and between WM-FNs and GM-FNs

\begin{tabular}{llll}
\hline WM-FNs & Functional networks & $\mathrm{t}$ value & $\mathrm{p}$ value \\
\hline Inferior fronto-occipital fasciculus WM-FN & Visual GM-FN & 4.085 & 0.007 \\
& Anterior cerebellar GM-FN & 3.266 & 0.048 \\
& Sensorimotor GM-FN & 3.252 & 0.048 \\
& Occipital WM-FN & 3.546 & 0.030 \\
& Pre/postcentral WM-FN & 3.205 & 0.046 \\
\hline
\end{tabular}

3 Abbreviations: mTBI, mild traumatic brain injury; HCs, healthy controls; FC, functional connectivity;

4 WM-FNs, white matter functional networks; GM-FNs, grey matter functional networks; WM-FN,

5 white matter functional network; GM-FNs, grey matter functional network;

6 Note: FDR corrected $\mathrm{p}<0.05$.

7 